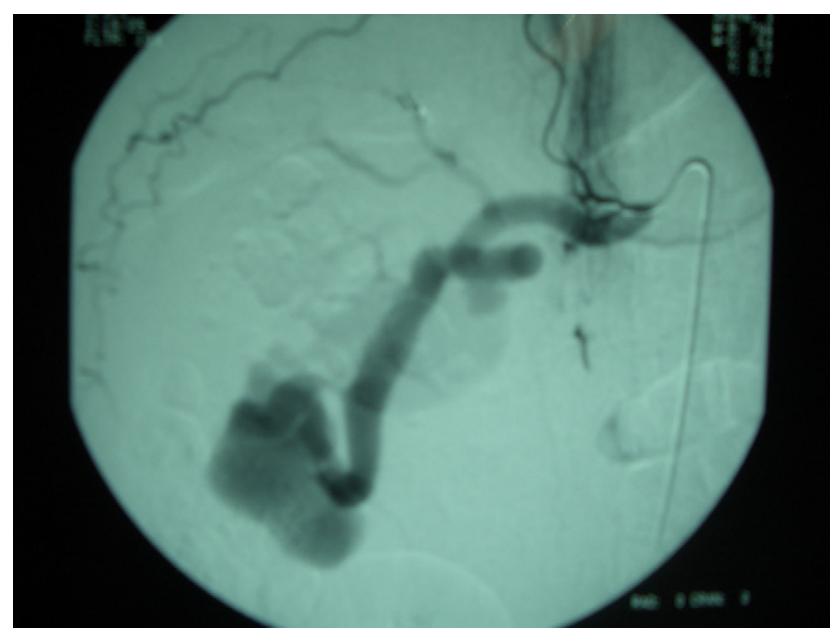

Figura 3 - Arteriografía renal con cateterización selectiva de la rama arterial renal superior comprobando que de dicha rama, se rellenan las cavidades aneurismáticas y el vaso aberrante ectásico.

Se realizó nefrectomía radical abierta, por vía anterior. Cursando con un postoperatorio sin complicaciones y actualmente el paciente se encuentra asintomático y con una función renal, dentro de los límites de la normalidad.

La anatomía patológica final fue informada como dilatación aneurismática arterial en forma de 2 lesiones de contenido hemorrágico que miden 3 centímetros de dimensión mayor cada una de ellas y se hallan localizadas en la región renal medial y en el polo inferior derecho. La arteria renal presenta cambios ateroescleróticos y aunque se halla dilatada en la porción inferior de su trayecto intrarrenal no se identifica microscópicamente clara conexión con las dilataciones aneurismáticas ni con las estructuras venosas. El resto del parénquima renal presenta múltiples focos de hemorragia intratubular e intraparenquimatosa con signos de sangrado antiguo. Nefroangioesclerosis. Uréter y pelvis se hallan dilatados y ocupados por material hemático.

En el estudio habitual de masas renales calcificadas, no hallamos descritos los aneurismas intrarrenales. Hasta el momento, se han descrito psedoaneurismas, microaneurismas, fístulas arteriovenosas y aneurismas de las arterias renales y sus segmentarias. Hasta la actualidad, y bajo nuestro conocimiento, no hemos encontrado descritas los aneurimas de arterias distales. Generalmente, las alteraciones vasculares, están acompañadas de clínica de hipertensión arterial ${ }^{1-4}$. En el caso que nos ocupa el único síntoma fue la hematuria macroscópica y el dolor lumbar de $48 \mathrm{~h}$ de evolución, probablemente la evolución fue mucho mayor, pues el paciente no relata que 15 años antes le diagnosticaron de calcificación renal derecha. Para un correcto abordaje de esta patología, es imprescindible una correcta valoración, y entre las pruebas que debemos incluir, la arteriografía renal, que será la prueba complementaria gold estándar. Respecto al abordaje quirúrgico, debemos tener un buen control de los grandes vasos y quizás la vía anterior sea una buena opción.

\section{B I B L I O G R A F Í A}

1. Porcaro AB, Migliorini F, Pianon R, Antoniolli SZ, Furlan F, De Biase $\mathrm{V}$, et al. Intraparenchymal renal artery aneurysms. Case report with review and update of the literature. Int Urol Nephrol. 2004;36:409-16. Review.

2. Akpolat T, Akkoyunlu M, Akpolat I, Dilek M, Odabas AR, Ozen S. Renal Behcet's disease: a cumulative análisis. Semin Arthritis Rheum. 2002;31:317-37. Review.

3. Jennette JC, Falk RJ. The pathology of vasculitis involving the kidney. Am J Kidney Dis. 1994;24:130-41. Review.

4. Dillon MJ. Renovascular hypertension. J Hum Hypertens. 1994;8:367-9. Review.

P. Navarro Medina ${ }^{\mathrm{a}, *}$, J.L. Artiles Hernández ${ }^{\mathrm{a}}$, C. Camacho García ${ }^{\mathrm{b}}$, A. Blanco Diez ${ }^{a}$, C. Jiménez García ${ }^{a}$ y N. Chesa Ponce ${ }^{a}$

aServicio de Urología, Hospital Universitario Insular de Gran Canaria, Gran Canaria, España

${ }^{\mathrm{b}}$ Servicio de Anatomía Patológica, Hospital Universitario Insular de Gran Canaria, Gran Canaria, España

*Autor para correspondencia.

Correo electrónico: patricionm@terra.es (P. Navarro Medina).

\title{
Nevus azul prostático. Un diagnóstico histológico poco frecuente
}

\section{Prostatic blue nevus. An infrequent histologic diagnosis}

\section{Sr. Director:}

Presentamos el caso de un paciente varón de 68 años, sin antecedentes de interés, con síndrome prostático de varios años de evolución, portador de sonda urinaria desde hacía 3 meses por episodio de retención aguda de orina, con exploración física normal y con tacto rectal con próstata lisa, elástica y sin nódulos duros. La analítica resultó normal, con 


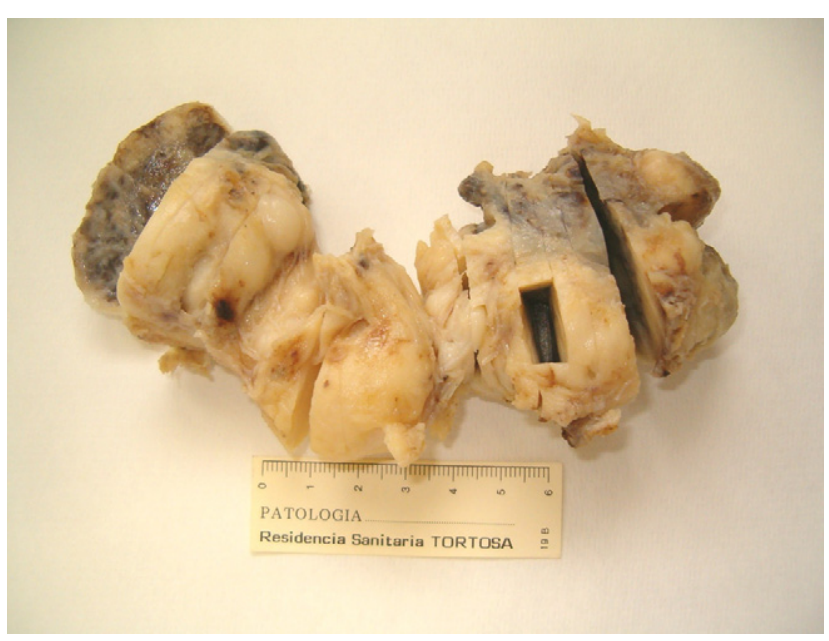

Figura 1 - Pieza de adenomectomía en la que se observan extensas áreas con pigmentación negroazulada que corresponden a la lesión. La superficie de corte muestra una marcada pigmentación negruzca.

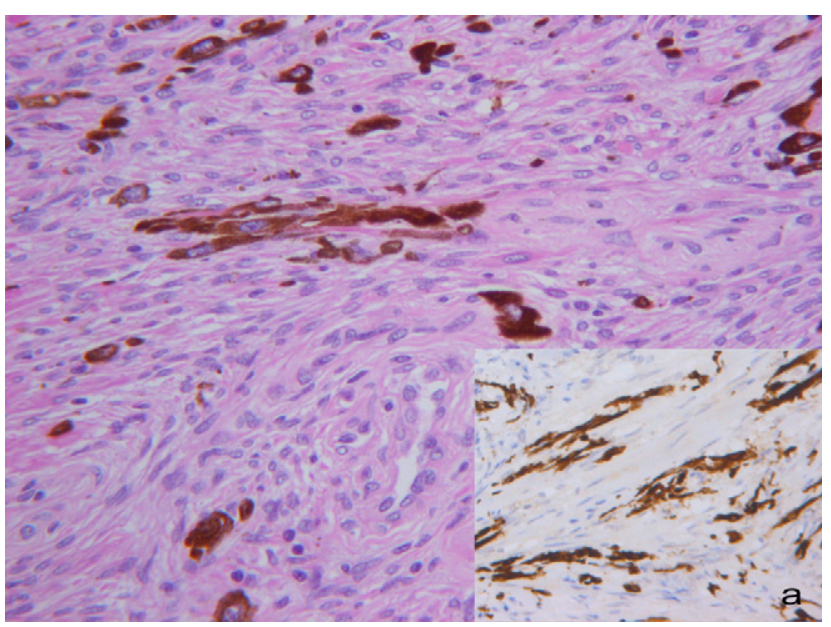

Figura 2 - Sección histológica teñida con hematoxilinaeosina donde se aprecia el pigmento melánico en el citoplasma de las células proliferantes que muestran morfología alargada y de aspecto dendrítico, sobre un estroma prostático sin anomalías adicionales y que no muestra atipia. HE $10 \times$. a) Se muestra la técnica immunohistoquímica de Melan-A que tiñe intensamente las células proliferantes del nevus azul de coloración marrón oscuro. Melan-A $10 \times$.

PSA de 2,06 ng/ml. La ecografía mostró riñones normales, vejiga anodina, volumen prostático de $100 \mathrm{~cm}^{3}$ y vesículas seminales normales y simétricas. Se realizó adenomectomía prostática retropúbica sin complicaciones. El estudio macroscópico mostró una pieza quirúrgica de $112 \mathrm{~g}$ y de $120 \times 45 \times 20 \mathrm{~mm}$, con superficies externa y de corte con extensas áreas negruzcoazuladas y con áreas de aspecto hiperplásico (fig. 1). En el estudio microscópico se observaron signos histológicos de hiperplasia nodular y en las zonas macroscópicamente negruzcas se observaron abundantes células alargadas u ovales, sin atipias, que adoptaban ocasionalmente morfología dendrítica (fig. 2) y presentaban pigmento citoplásmico de color marrón oscuro y aspecto melánico. La distribución de la celularidad pigmentada era irregular y se hallaba situada exclusivamente en el estroma, sin apreciarse pigmentación del epitelio glandular. La tinción de Perls para el hierro fue negativa. Las técnicas de inmunohistoquímica para la proteína S-100, HMB-45 y Melan-A (fig. 2a) resultaron positivas. El diagnóstico anatomopatológico fue de nevus azul (NA) prostático (NAP).

El NA es una neoplasia benigna típica de la piel caracterizada por una proliferación de melanocitos, de morfología dendrítica o fusiforme y de citoplasma con pigmento melánico ${ }^{1}$. La localización extracutánea del NA es infrecuente, habiéndose descrito su presencia en 2 localizaciones urológicas: el cordón espermático y la próstata ${ }^{2}$. Son muy escasas las publicaciones de NA que afecte a la próstata. Tanto su patogénesis como su incidencia se desconocen, aunque probablemente el origen de estas células se encuentra en la cresta neural. Su presencia no implica sintomatología clínica específica y se trata de una lesión benigna en la que no se ha descrito degeneración a melanoma maligno $^{3}$. Su hallazgo es casual, generalmente tras el estudio histopatológico por hiperplasia adenomatosa benigna, aunque también se ha descrito su asociación con adenocarcinoma prostático ${ }^{4}$. Existe controversia y cierta confusión en la terminología empleada por los distintos autores sobre las lesiones pigmentarias prostáticas. La definición más ampliamente aceptada de NA es aquella en la que el pigmento melánico se localiza en las células del estroma prostático, mientras que el término melanosis se reserva para los casos en los que el pigmento se localiza únicamente a nivel epitelial. A pesar de ello, muchos autores usan indistintamente estos dos términos ${ }^{5}$. Actualmente se admite que solo tienen capacidad para la melanogénesis las células del estroma prostático y no las células epiteliales, y que la melanina pasa del estroma al epitelio de forma pasiva ${ }^{6}$. En el diagnóstico diferencial del NAP deben incluirse las pseudomelanosis o lipofuscinosis prostáticas en las que el citoplasma de las células epiteliales prostáticas contienen gránulos parduzcos o hematoxilínicos de lipofuscina. Este se incrementa de forma considerable con el envejecimiento celular y las agresiones (infecciones sistémicas, locales o la toma de fármacos); se deposita en la mayoría de los órganos, en los epitelios normales, en los hiperplásicos, en los neoplásicos y puede también depositarse en el estroma; probablemente es el responsable de muchas de las melanosis prostáticas que no se encuentran totalmente documentadas en la literatura médica. Otro diagnóstico diferencial histopatológico que debe incluirse son las lesiones prostáticas producidas por depósito de hierro (hemorragias, infartos, abscesos y hemocromatosis primaria o secundaria). De vital importancia es incluir el diagnóstico diferencial histológico con el melanoma maligno prostático primario o metastático ${ }^{7}$, que representa menos del $1 \%$ de todos los melanomas en humanos; su diagnóstico es difícil y comporta un tratamiento quirúrgico agresivo ${ }^{8,9}$, habiéndose publicado muy pocos casos y la mayoría secundarios, originados en la uretra prostática o por metástasis ${ }^{7}$. Finalmente, en cuanto a la conducta por seguir tras el diagnóstico de NAP, dada la ausencia de casos descritos en la literatura médica de degeneración maligna y la extrema rareza de la malignización de esta entidad en la piel, no parecen aconsejables revisiones o controles periódicos adicionales a los recomendados por la 
patología de base que llevó al estudio histológico y a su diagnóstico. En resumen, hemos presentado un nuevo caso de NAP, descubierto incidentalmente en el estudio anatomopatológico de una pieza de adenomectomía prostática con una hiperplasia benigna de próstata y hemos discutido los principales diagnósticos diferenciales anatomopatológicos, entre los que destaca el diagnóstico diferencial con el melanoma maligno primario o secundario.

B I B L I O G R A F Í A

1. Kerl H, LeBoit PE, Bastian BC. Pathology and genetics of skin tumors. En: LeBoit PH, Weedon D, Sarasin A, editors. WHO. Lyon: IARC Press; 2006. p. 95.

2. González-Campora R, Galera-Davidson H, Vazquez-Ramírez FJ, Díaz-Cano S. Blue Nevus: Classical types and new related entities. A differential diagnostic review. Pathol Res Pract. 1994;190:627-35.

3. Cuervo Pinna C, Godoy Rubio E, Parra Escobar JL, Sánchez Blasco E, Valverde Valverde J, Moreno Casado J. Nevus azul prostático. Estandarización de la terminología de las lesiones pigmentadas de la próstata. Actas Urol Esp. 2001;25:245-7.

4. Klock C, Gomes R, Joao M, Netto G. Prostate melanosis associated with acinar adenocarcinoma. Int J Surg Pathol. 2008.
5. Redondo Martínez E, Rey López A, Díaz Cascajo C. Nevus azul de la próstata. Diagnóstico diferencial de las lesiones pigmentadas prostáticas. Arch Esp Urol. 1998;51:286-9.

6. Vesga Molina F, Acha Pérez M, Llarena Ibarguren R, Pertusa Pena C. Nevus azul intraprostático. Arch Esp Urol. 1995;48: 985-6.

7. Wang CJ, Chen YT, Shun CT, Lai MK. Primary malignant melanoma of the prostate. J Urol. 1995;154:1865.

8. Wong J, Wise GJ, Clark B. Malignant melanoma of the prostate: A case report. Can J Urol. 2008;15:4027-9.

9. Wang CJ. Followup of primary malignant melanoma of the prostate. J Urol. 2001;166:214.

\section{J. Marti-Mestre ${ }^{\mathrm{a}}$, D. Cañis-Sánchez ${ }^{\mathrm{a}}$, J.-M. Jaén-Martínez ${ }^{\mathrm{b}}$ y R. Bosch Princep ${ }^{\mathrm{b}, *}$}

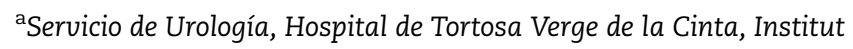
d'Investigació Sanitaria Pere Virgili, Tortosa, Tarragona, España ${ }^{\mathrm{b}}$ Servicio de Anatomía Patológica, Hospital de Tortosa Verge de la Cinta, Institut d'Investigació Sanitaria Pere Virgili, Tortosa, Tarragona, España

*Autor para correspondencia.

Correo electrónico: rbosch.ebre.ics@gencat.cat

(R. Bosch Princep).

\section{Seminoma clásico testicular que inicia como abdomen agudo. A propósito de un caso}

\section{Classic testicular seminoma that initiates as acute abdomen. A case report}

\section{Sr. Director:}

La semiología del tumor testicular ya está descrita de forma extensa en la literatura médica, y en la mayor parte de los pacientes se circunscribe al escroto. El caso que nos atañe presenta una forma atípica de inicio, por lo que creemos que su lectura puede resultar de interés y constituye un ejemplo más de que la exploración física completa es crucial en la evaluación de un paciente.

Acude a nuestro servicio de Urgencias un varón de 39 años, procedente del este de Europa, sin antecedentes de interés. Refiere dolor tipo cólico transfixiante de predominio en el hemiabdomen inferior, en la fosa ilíaca izquierda, que ha sido refractario a analgésicos habituales. Niega traumatismo previo u otra sintomatología urológica.

A la exploración física, presenta una masa en el vacío-fosa ilíaca izquierda, dolorosa a la palpación. El teste derecho es normal, se palpan dos nódulos de consistencia pétrea en el teste izquierdo.

En el hemograma se observa una discreta leucocitosis (10.900 L/Mcl), con una neutrofilia del 90,7\%, una linfopenia de
6.300 Leucocitos/Mcl y una trombocitosis de 464.000; la bioquímica no presenta alteraciones reseñables y en la gasometría se observa una alcalosis respiratoria con un $\mathrm{pH}$ de 7,62. A la vista de estos hallazgos, se realiza un estudio ecográfico abdominal, en el que se visualiza una imagen compatible con un conglomerado adenopático retroperitoneal precavoaórtico de aproximadamente $11 \times 6 \mathrm{~cm}$, siendo el nódulo mayor paraaórtico de $6 \times 5 \mathrm{~cm}$. En el lóbulo hepático derecho se observa una lesión focal sólida hipoecogénica de $8 \mathrm{~mm}$. La ecografía escrotal muestra el teste derecho normal $\mathrm{y}$, en el izquierdo, microlitiasis y dos nódulos hipoecoicos de 14,3 y de $8,5 \mathrm{~mm}$, y la presencia de una masa de $4 \mathrm{~cm}$.

Se somete al paciente a orquiectomía radical izquierda, sin incidencias. El diagnóstico anatomopatológico es el siguiente: seminoma clásico con intensa reacción sarcoidea y extensión a las cubiertas testiculares, al epidídimo y a la parte proximal del cordón (fig. 1); áreas de Cis; lesiones esclerosadas compatibles con áreas de tumor quemado y vasculitis granulomatosa de vasos del epidídimo e intratumorales (seminoma clásico pT3).

La TC descarta la existencia de enfermedad nodular torácica y de lesiones en el parénquima pulmonar. El hígado, 